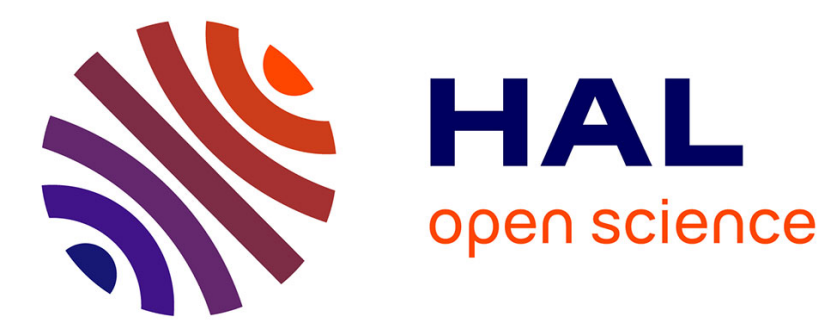

\title{
Un nouveau fragment d'inscription chrétienne à Briord (Ain)
}

Françoise Descombes, Roger Perraud

\section{To cite this version:}

Françoise Descombes, Roger Perraud. Un nouveau fragment d'inscription chrétienne à Briord (Ain). Gallia - Fouilles et monuments archéologiques en France métropolitaine, 1988, 45, pp.211-212. 10.3406/galia.1987.2888 . hal-01939610

\section{HAL Id: hal-01939610 https://hal.science/hal-01939610}

Submitted on 14 Jan 2020

HAL is a multi-disciplinary open access archive for the deposit and dissemination of scientific research documents, whether they are published or not. The documents may come from teaching and research institutions in France or abroad, or from public or private research centers.
L'archive ouverte pluridisciplinaire HAL, est destinée au dépôt et à la diffusion de documents scientifiques de niveau recherche, publiés ou non, émanant des établissements d'enseignement et de recherche français ou étrangers, des laboratoires publics ou privés.

\section{(이) $\$$}

Distributed under a Creative Commons Attribution - NonCommercial - NoDerivatives| 4.0 


\title{
UN NOUVEAU FRAGMENT D'INSCRIPTION CHRÉTIENNE À BRIORD (AIN)
}

\author{
par Françoise DESCOMBES avec la participation de Roger PERRAUD
}

M. Perraud, fouilleur du chantier des Plantées à Briord $^{1}$, et son équipe, ont eu l'occasion de trouver à plusieurs reprises de nouvelles inscriptions sur ce site, qui était déjà connu par des découvertes anciennes, avec quelques textes tardifs fort intéressants du point de vue épigraphique et même historique $^{2}$. Il y a 25 ans, MM. Perraud et Marquié m'avaient confié des documents que j'avais présentés à la Société Nationale des Antiquaires de France $^{3}$. Tout récemment, M. Perraud avait l'amabilité de me communiquer à nouveau un modeste fragment mis au jour fortuitement, qui ne révolutionnait pas nos connaissances des séries épigraphiques de Briord mais qu'il fallait, de toute évidence, publier. J'ai cru bien faire de demander à Françoise Descombes, auteur du Recueil des Inscriptions Chrétiennes de la Gaule, $\mathrm{XV}$, de rédiger une "fiche", comme s'il s'agissait d'une publication de corpus.

\section{Noël Duval}

1 A. Grange, H. Parriat, R. Perraud, La nécropole gallo-romaine et barbare de Briord, Ain, La Physiophile, Montceau-les-Mines, 58, 1963 et 62, 1965. Cf. M. Le Gray, Informations archéologiques, Gallia, 24, 1966, p. 485-487 (voir aussi, La Physiophile, 52, 1960; 60, $1964 ; 75,1971 ; 79,1973$; 88, 1978). En dernier lieu, H. Parriat, R. Langrand et $R$. Perraud, La nécropole gallo-romaine et mérovingienne des Plantées à Briord (Ain). Synthèse et résultats des fouilles de 1958 à 1973, La Physiophile, 92, 1980, p. 16-50; La nécropole gallo-romaine et mérovingienne des Plantées à Briord (Ain). Les Plantées-nord. Synthèse et résultats des fouilles de 1959 à 1981, La Physiophile, 104, 1986 (Iommage à A. Rigoulot), p. $51-67$.

2 F. Drscombes, Recueil des Inscriptions Chrétiennes de la Gaule (R.I.C.G.), XV, 1984, no 258-275, p. 663-709. Cf. introduction, $\S 23$ p. 24-26 et $\S 71-2$ p. 43.

3 N. Duval, Les dates de quelques inscriptions chrétiennes de Briord, Bulletin de la Société Nationale des Antiquai-
Briord (canton de Lhuis, Ain).

Fragment trouvé vers 1980 par un jeune garçon, Sylvain Laroche, à l'intérieur du tunnel-aqueduc de Briarette, près de l'entrée o., qui domine la nécropole gallo-romaine et mérovingienne des Plantées. Le fragment se trouvait au-dessus d'une tranchée creusée récemment pour une canalisation d'eau, et pourrait avoir fait partie des matériaux de comblement de la tranchée; il est donc de provenance incertaine.

Conservé au Musée de Briord.

Renseignements et photographie fournis par M. R. Perraud, Conservateur du Musée.

Marbre brisé de tous côtés, sauf à gauche.

H. 16 ; I. 19 ; ép. 3,5 à $5,7 \mathrm{~cm}$.

Lettres : 3 à $3,5 \mathrm{~cm}$ environ aux quatre premières lignes, plus petites à la dernière ligne (2,5 cm env.).

Une marge d'environ $6 \mathrm{~cm}$, à gauche, est occupée dans sa partie supérieure par deux longs traits obliques pour lesquels je ne vois pas d'explication satisfaisante : ces raies doivent être récentes (coups d'outil?).

Hoc ou $I(n)$ oc? t![umulo requiescit? in pace]

Euch/erius/a (ou -arius/a) ... qui/ae ui]-

xil a[nnos...] ou [nnis ou [nnus...]

obiit [in pace...? ianu- ou febru-]

arịas $[\ldots]$

Le fragment n'offre pas de difficulté de lecture proprement dite. Seuls font problème les traits déjà

res de France, 1962, p. 63-73; Nouveaux fragments d'inscription du viI ${ }^{\mathrm{e}}$ s. trouvés à Briord (Ain), ibid., 1964, p. 77-84. Cf. P. Wuilleumier, Inscriptions latines des Trois Gaules, XVII supplément à Gallia, réédition C.N.R.S. 1981, p. 120, n 304 ; Année épigraphique, 1965, p. 41 no 141 , p. 110 nos 339 bis et ter. 


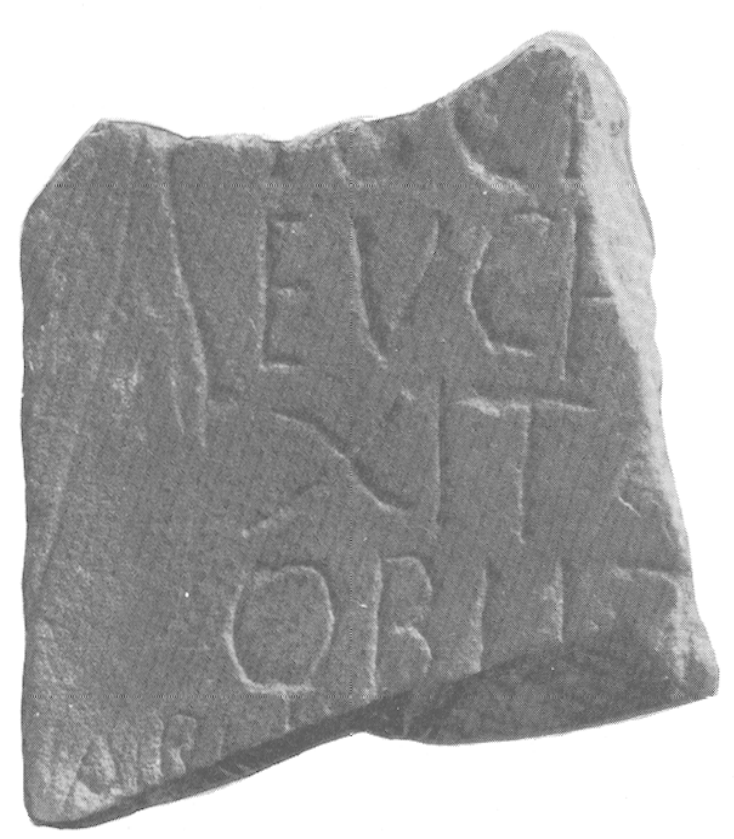

signalés dans la marge de gauche et le début de la 1. 1 (voir ci-dessous).

On distingue à la fin de la 1.1 une haste verticale et la partie gauche du linteau d'un $T$; à la fin de la 1.3 le A de $a / n n o s ; a u$ début de la 1.5 , avant la terminaison du nom du mois -arias, un trait vertical ou légèrement oblique; faute d'avoir vu la pierre elle-même, il est difficile de dire s'il s'agit d'un $V$ ou d'un trait parasite (il paraît moins profondément gravé), comme il y en a dans la partie supérieure. Le sommet des lettres $A R I A$, et même $S$, est encore nettement visible.

Lettres de facture médiocre. Les $\mathrm{A}$ à traverse brisée (l. 3 et 5 ), les $X$ dont une barre est ondulée (l. 3 ), le 0 circulaire (1. 4) indiquent la fin du ve ou le premier tiers du vi ${ }^{\mathrm{e}}$ s. (voir RICG, XV, Intr., § 132). On notera en outre les deux $\mathrm{C}$ des $\mathrm{l} .1$ et 2 , le second à peu près "classique", le premier ouvert en bas et comme basculé en arrière.

Il s'agit là $d u$ fragment d'une inscription funéraire au formulaire tout à fait banal.

L. 1 : une seule incerlilude subsiste au début de la première ligne; on lit nettement les lettres $O C T$, et on reconnaît donc la formule in hoc tumulo requiescit, suivie peutêtre de in pace (la place semble manquer pour bonae memoriae). Or on ne voit plus, à gauche du $O$, que la partie inférieure d'une seule haste verticale, et la place manque, si l'on tient compte de l'alignement, pour-les quatre hastes attendues (deux seulement si on admet la graphie oc pour hoc (cf., à Briord, RICG, $X V, 270$ ). Peut-être s'agit-il d'une erreur de graphie $\mathrm{du}$ lapicide, comme on en trouve dans quelques cas isolés (cf. RICG, XV, Intr., § 149).

L. 2-3 : la formule indiquant l'âge comportait le mot a[nnos], a[nnis] ou a[nnus] : les trois formes se trouvent dans les inscriptions des ve et $\mathrm{vi}^{\mathrm{e}} \mathrm{s}$. de Briord (RICG, $\mathrm{XV}$, $\mathrm{n}^{\text {os }}$ 258-262). La forme annos est toutefois la plus courante.

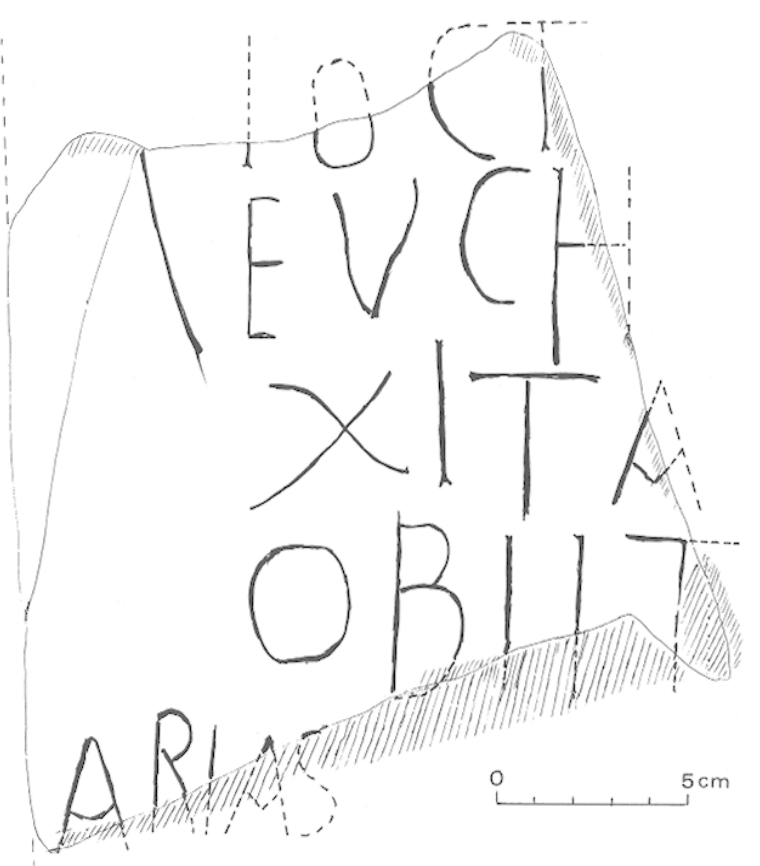

L. 4-5 : la formule introduisant la date de la mort comportait sans doute l'expression in pace, habituelle dans la série la plus ancienne de Briord. Le nom du consul indiquant l'année a disparu.

Euch $[. . . /$, à la l. 2, est le début du nom du défunt ou de la défunte, Eucharius/a, Eucherius/a. Les deux formes, toutes deux dérivées du grec $\varepsilon \tilde{\chi} \chi \alpha$ p เs, "gracieux", "élégant", sont bien attestées. Eucharius et Eucherius ont été les noms de plusieurs évêques de Gaule (voir Diehl III, p. 54; RICG, I, 19; RICG, $\mathrm{XV}, 2)$.

On a trouvé jusqu'ici à Briord deux séries d'inscriptions chrétiennes, l'une du ve et du début du $\mathrm{vI}^{\mathrm{e}}$ s., provenant du lieu-dit Le Pontonnier, au n.-o. de l'église, l'autre du vir ${ }^{\mathrm{e}}$ s., dans laquelle se rangent quelques fragments trouvés récemment aux Plantées, à l'e. de Briord (RICG, XV, Intr., §23).

Le fragment trouvé dans le tunnel de Briarette appartient, d'après son écriture, à la série la plus ancienne. Mais rien ne permet d'affirmer qu'il a la même origine géographique: le lieu de trouvaille n'est pas significatif ; le formulaire initial est différent de celui des épitaphes du Pontonnier et peutêtre légèrement plus tardif (on trouve hic requiescit dans la sẹrie du Pontonnier, et (in) hoc tumulo requiescit, avec peut-être in pace, ici ; voir $R I C G, \mathrm{XV}$, Intr., $\S 139,141)$. On ne peut exclure par ailleurs l'existence possible d'épitaphes antérieures au viI ${ }^{\mathbf{e}} \mathrm{s}$. sur le site des Plantées, puisque la nécropole existe depuis l'époque gallo-romaine (voir La Physiophile, Montceau-les-Mines, 58, 1963, et 92, 1980).

\section{Françoise Descombes}

N.B. - Sur le dessin, un trait a été omis à l'extrémité gauche de la dernière ligne. 